\section{Strömungsverlauf und Mischzeiten in axialen Strahlmischern *}

\section{Imre Rácz und Jan Groot Wassink * *}

Unter einem Strahlmischer versteht man im allgemeinen ein Mischsystem, wobei dem Mischbehälter mit Hilfe einer Pumpe ein bestimmter Volumenstrom entnommen und durch eine Düse wieder zurückgeführt wird. Durch Impulsaustausch zwischen Strahl und Behälterinhalt wird der Behälterinhalt in Bewegung gesetzt.

Beim Homogenisieren löslicher Flüssigkeiten in Großbehältern werden neben Propellerrührern auch Strahlmischer eingesetzt (z. B. beim Homogenisieren von Benzin-Fraktionen); sie zeichnen sich durch konstruktiv einfachen Aufbau aus. Strahlmischer eignen sich auch zur Anwendung in kleinen Behältern. Thre noch relativ geringe Verbreitung in der chemischen Technik beruht unter anderem auf der Unkenntnis entsprechender Dimensionierungsmöglichkeiten.

In der Literatur wurden bis jetzt nur wenige experimentell gewonnene Untersuchungsergebnisse über Strahlmischer mitgeteilt [1-4]. Diese Ergebnisse sind nur schwer miteinander zu vergleichen, weil bei den Untersuchungen verschiedenartige Meßmethoden verwendet wurden, der Endwert der Mischzeit nicht eindeutig festgelegt wurde und die Geometrie der Mischgefäße nicht die Gleiche war.

Bei unserer Forschungsarbeit wurde ein runder Strahl axial in das zylindrische Mischgefäß eingeführt, eine Geometrie, die bisher noch nicht untersucht worden ist, obwohl eine weitgehende Übereinstimmung besteht zwischen dieser Geometrie und der Geometrie des Standard-Rührgefäßes, ausgestattet mit einem axialen Rührer, das in den letzten Jahrzehnten eingehend untersucht worden ist [5].

Zur Untersuchung der Strömungsprofile wurde eine photographische Methode verwendet, wobei die folgenden Einflußgrößen geändert wurden: Behälterdurchmesser, Behälterhöhe, Düsendurchmesser, Bodenabstand der Düse, Düsenausflußgeschwindigkeit und die Lage der Ausflußöffnung aus dem Behälter. In allen untersuchten Fällen entstand etwa das gleiche Strömungsprofil (Abb. 1):

Auf dem Boden des zylindrischen Behälters entsteht ein Wirbelring, dessen Höhe gleich dem halben Durchmesser ist. Wenn die Höhe des Behälters größer ist als der halbe Durchmesser des Behälters, dann sind die Flüssigkeitsgeschwindigkeiten oberhalb der Düsenöffnung sehr klein. In diesem Fall ist der Wirbel nicht stabil, er löst sich periodisch auf.

Zur Bestimmung der Mischzeit $t_{\mathrm{m}}$ wurde der Konzentrationsverlauf' in der Umwälzleitung gemessen, nachdem in dem Strahlmischer eine bestimmte Menge KCl-Lösung injiziert worden war.

- Vortrag auf dem Jahrestreffen der Verfahrens-Ingenieure, 2. bis 4. Oktober 1973 in Berlin.

** Ir. I. G. Rácz, Prof. ir. drs. J. Groot Wassink, Technische Universität Twente, Enschede, Postfach 217, Niederlande.
Bei den Mischzeitmessungen wurden die folgenden Einflußgrößen geändert: Behälterdurchmesser $D_{T}$, Behälterhöhe $H_{\mathfrak{T}}$, Düsendurchmesser $D_{\mathrm{n}}$, Bodenabstand der Düse, Ausflußgeschwindigkeit aus der Düse $V_{n}$, Dichte der Flüssigkeit, Zähigkeit der Flüssigkeit. Die statistische Auswertung der Messungen ergab für die dimensionslose Mischzeit $T$ die folgenden Beziehungen :

für Behälter mit $H_{\mathbf{T}}=D_{\mathrm{T}}$ :

$T=\frac{t_{\mathrm{m}} V_{\mathbf{n}}}{D_{\mathrm{n}}}=1,1\left(\frac{D_{\mathrm{T}}}{D_{\mathrm{n}}}\right)^{2,75}$,

für Behälter mit $H_{\mathrm{T}}=1 / 2 D_{\mathrm{T}}$ :

$T=\frac{t_{\mathrm{m}} V_{\mathbf{n}}}{D_{\mathrm{n}}}=2,6\left(\frac{D_{\mathbf{T}}}{D_{\mathbf{n}}}\right)^{2,0}$

mit einer Abweichung $\leq 10 \%$ des gemessenen Wertes.
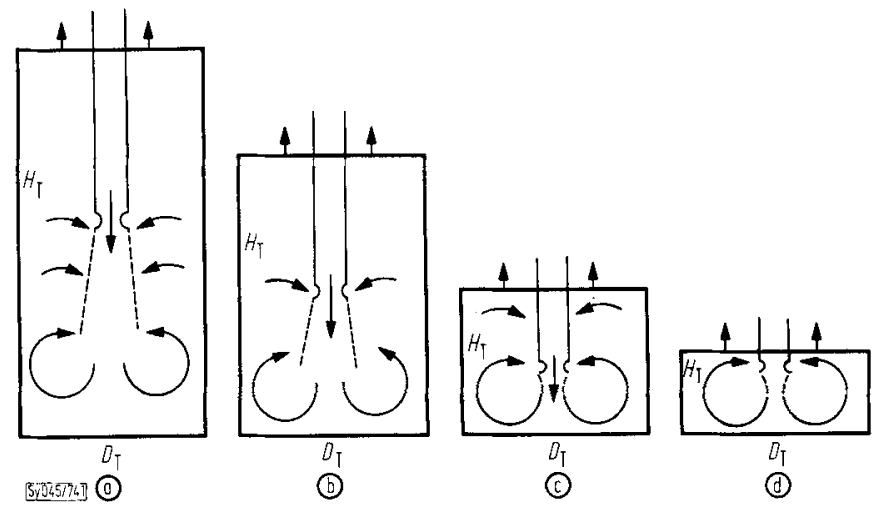

Abb. 1. Strömungsprofile in den Behältern.

$\mathrm{a}: H_{\mathrm{T}}=3 D_{\mathrm{T}} ; \mathrm{b}: H_{\mathrm{T}}=2 D_{\mathrm{T}} ; \mathrm{c}: H_{\mathrm{T}}=D_{\mathrm{T}} ; \mathrm{d}: H_{\mathrm{T}}=1 / 2 D_{\mathrm{T}}$.

Ein Vergleich dieser Ergebnisse mit den Ergebnissen von Propellerrührern aus der Literatur ergibt, daß der axiale Strahlmischer bezüglich der Mischzeit und des spezifischen Energieverbrauchs unter gewissen Bedingungen eine günstigere Wirkung beim Homogenisieren löslicher Flüssig. keiten als der Propellerrührer aufweist.

Eingegangen am 16. November 1973

Literatur

[1] H. Fossett, L. F. Prosser, J. Proc. Inst. Mechan. Engng. 160, 224 [1949].

[2] E. A. Fox, V. E. Gex, A.I.Ch.E. Journal 2, 539 [1956].

[3] J. G. van de Vusse, diese Zeitschr. 31, 583 [1959].

[4] N. Okita, Y. Oyama, Kagaku Kogaku (Abridged Edition) 1, 94 [1963].

[5] R.M. Voncken, Circulatiestroming en menging in geroerde vaten Universitaire pers, Rotterdam 1966.

Schlüsselworte: Homogenisieren, Strahlmischer, Strömungsprofile, Mischzeiten.

Das vollständige Manuskript dieser Arbeit umfaßt 28 Seiten mit 10 Abbildungen, 4 Tabellen und 7 Literaturzitaten. Es ist als Photokopie oder Mikrofiche MS 045/74 erhältlich. Bestellkarten finden Sie in diesem Heft. 